\title{
ENERGY PELLETS
}

\author{
Leif Jilkén \\ University of Kalmar, Sweden
}

\begin{abstract}
Thermoplastic waste from industrial enterprises and commerce constitutes a considerable potential for conservation of energy resources.
\end{abstract}

Another energy resource is organic fibres, such as woodfibres from waste. By ad ding waste fibres and thermoplastic waste in a composite ENCO we have got a new fuel pellet/material.

Thermoplastic waste from polyethylene (P E) and polypropylene (PP) together with woodfibres are used in ENCO to reach a high energy density.

\section{Keywords}

Energy Pellets, Recycling, Environmental Driven Technology, ENCO, Thermoplastic waste, Wood- fibres.

\section{History}

Since immemorial times we have lived from what the forrest had been given us, we have built our houses, furnish from wood and heated up our houses with firewood. Even today it is our major renewable resource, the annually growth is bigger than we annually felling in Sweden.

The great changeover procedure who has started up within the heating area according to a decision in the Swedish Parliament demands new system solutions. The dependence of electricity shall de- crease, but the environment must not deteriorate. If it is possible to use one or more recyclable materials, domestic rawmaterials ought to be used.

The major advantage with bio-fuels is that they give no net contribution to the greenhouse effect and discharges of acidification materials.

\section{Aim}

Sweden has an enormous great wealth in its abundance of bio-fuel. This project aim to process bio-fuel mixed with recycled thermoplastic waste, in an energy composite, such that moisture can' $t$ penetrate into the bio-fuel during the storing time or the transports. Moisture and unprotected bio- fuel drastically decrease the useable energy value, see figure 1 , furthermore the moisture creates the necessary conditions for mould growth with major health hazards. 


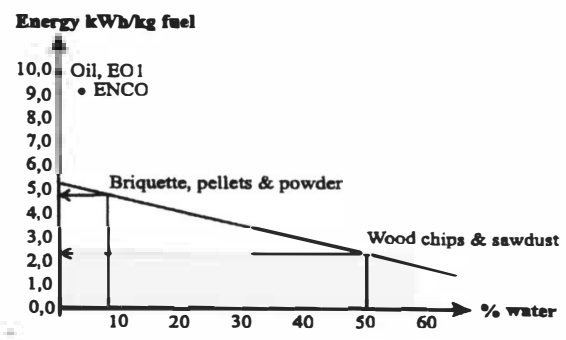

Figure 1. The influence of water on energy density.

\section{Statement of the art}

Woodpellets had been developed to be an interesting fuel for heating of area of one-family houses because it is simple to transport and store. Figure 2 shows the pellets consumption in Sweden.

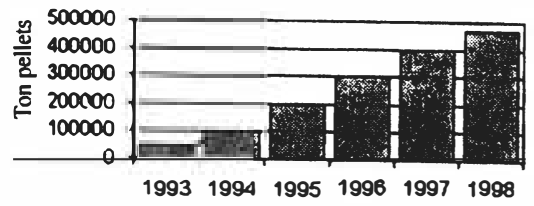

Figure 2. Pellets consumption in Sweden

By-products from sawmills and woodworking industry, sawdust and bark, consist of rawmaterial in the processing of bio-pellets.

During drying, grinding and compressing has the raw sawdust and bark, without fixing agents re- fine to finished bio-pellets with energydensity $4.7 \mathrm{kWh} / \mathrm{kg}$. The pellet density is about 650 $\mathrm{kg} / \mathrm{m} 3$ with $92 \%$ dryness.

\section{Components in ENCO}

\subsection{Organic fibres -woodfibres}

Wood is a natural material built up for fulfil some functions with in the growing or living tree. Its tubular structure is a result or that liquid and feeding stuff are able to transport in different directions. The fibres or tubular thickness differ depend on when it has growned, autumn/winter thick walls (high mechanical stresses) or spring/summer thin walls (main function is to transport liquids), this gives a large variation in density in different parts of the tree. Since a huge amount of liquid transport will go on continuously from the roots to the top of a tree (500 litres/day) are 
the helical fibre mainly parallel to the direction of the tree, this gives a high anisotropy in a piece of wood. See figure 3.

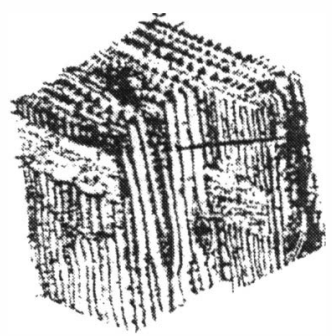

Figure 3. A piece of wood showing the fibre construction.

An interesting observation is that the compactdensity (the density reaching if graining the woodfibres) is relative independent of different types of trees. See figure 4.

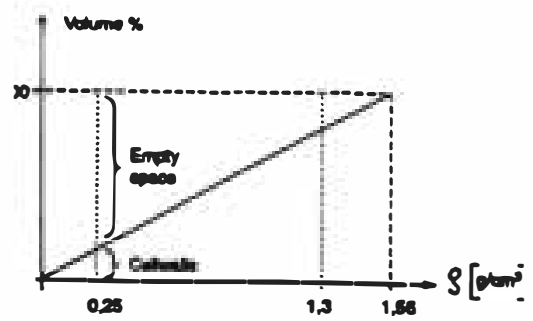

Figure 4. "Empty space" depending on density

The cellwalls are built up of cellulose, hemi cellulose and lignin. From a mechanical point of view the construction is, in the wall, such that the mechanical loads transforms to stresses in the chains of cellulose molecules, which are embedded in lignin. The cellulose molecules make helicals configuration there the angel of climb can varies. The cell walls include a lot of voids of different shape and size, from a few Ångström $(\AA)$ up to hundreds Ångström in size. This explains why wood has so enormous "inner surface" of about 400 square metres in one gram of dry wood; this gives possibilities to enclose other material as water, oil and polymers.

\subsection{Polymers}

Biological polymers have been around in a natural form since life began and play an extremely important role in plants and animals life, and include starch, proteins, lignin, cellulose, collagen, silk and natural robber.

Use synthetic polymers, has increased enormously over the past few decades, because of their relative cheapness, easy to fabricate, low density, chemical inertness and high electrical 
resistivity. Synthetic polymers derive mainly from oil based products and include polyethylene PE, polypropylene FP, polyamide PA (nylon), phenolics, synthetic robber. ..

We can separate polymers in two different boxes, see figure 4 , depending on type of architecture on polymer molecules.

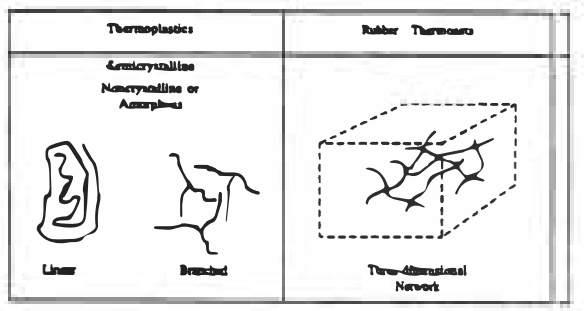

\section{Figure 5. Three different types of polymer molecules}

Polymer products are made up of long molecules. A typical sample of polyethylene may have molecules which contain an average 50000 atoms and would be $25000 \AA$ long $\left(1 \AA=10^{-10} \mathrm{~m}\right)$ The presence of these macromolecules has a dominant effect up on the properties of polymetric material.

Thermoplastics, which are often referred to just as "plastics" are linear of branched polymers, see figure 5, which can be melted upon the application of heat. They can be moulded and remoulded using conventional techniques and now make up the largest bulk of polymer used. Thermoplastics can be sub-divided into those, which partly crystallise on cooling (semi crystalline) and those, which do not and are normally used as polymer glasses, non crystalline (amorphous). Over eighty percent of plastics are thermoplastic, the main ones being polyethylene PE, polypropylene PP, polyvinylchoride PVC and polystyrene PS; all the other thermoplastics only account for about seven percent of the thermoplastics market.

Thermo sets and robber are heavily cross-linked polymers, which are normally rigid and intractable. The consist of dense three-dimensional molecular network, degrade rather than melt on the application of heat.

\section{How to mix together reusable thermoplastics with short fibres from different types of wood.}

The free volume is the space in a solid or liquid sample, which is not occupied by polymers molecules i.e. the "empty-space" between molecules. In the liquid state it is supposed that the free volume is high so molecular motion is able to take place relatively easily because the unoccupied volume allows the molecules space to move and to change their conformation freely. As the temperature of the melt is lowered the free volume will be reduced, see figure 6 . Under the glass - transition temperature region, $\mathrm{T}_{\mathrm{g}}$ is polymers so called "frozen", 
Spec. volume $\mathbf{V}$

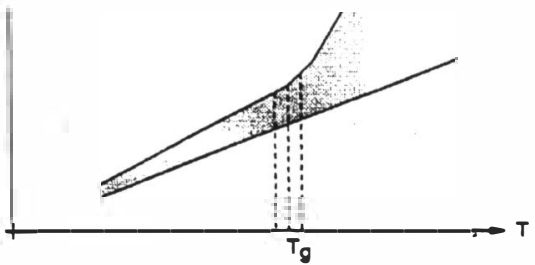

Figure 6. Schematic illustration of the variation of specific volume $V$, of a polymer with temperature $T$. The free volume is represented by the shaded area.

Mixing the composite should be done above the transition temperature $T_{\mathrm{g}}$ because the molecules move relativel! free there. The fibres we choose should be recoverable and damage the production equipment as little as possible, therefore we select short organic woodfibres. This fibre has an "inner surface" which gives a good coupling effect. [1, 2, 3, 4, 5, 6]

\section{Results and summary}

The energy composite (ENCO) can be put together in different ways, which results in different energy density. The ENCO draft reduces the dust spreading during the transportation and storing time.

Introductory tests of ENCO (appendix) indicate that the energy density is higher than for traditional wood pellets $\sim$ see figure 1 [7].

ENCO is regional produced and consuming, which results in low environmental impact in comparison with for instant oil and other fossil fuels. The relatively small amount of ashes from combustion of ENCO can be spread in the own garden. The ashes give both a fertiliser effect and raise the pH-value.

\section{References}

[1] Jilkén, L. From an idea to a recycling industry. Seminar on waste management and the environment

[2] 4'h Intermational Conference on Woodfiber-Plastic Composites, Madison, Wisconsin, May

[3] Pat. SE 9503190-2 [4] W093/25609 [5] W094/09959

[6] Jilkén L, Recoverable Composite Material made of Thermoplastic Waste combined with

Organic Fibres. Eco Design '99' -First International Symposium on Environmental Conscious Design and Inverse Manufacturing February 1-3, 1999, Tokyo, Japan

[7] Berglin O, Analys av en fraktion av EnKo, Högskolan i Kalmar,980603. 